MATEC Web of Conferences 11,03017 (2014)

DOI: 10.1051/matecconf/20141103017

(C) Owned by the authors, published by EDP Sciences, 2014

\title{
Piégeage des métaux lourds dans le mortier à court terme
}

\author{
Kalai Latifa $^{1}$, Rouis Mohamed Jamel ${ }^{1}$, Mnif Thameur ${ }^{2}$ \\ ${ }^{1}$ Université de Sfax, Ecole nationale des ingénieurs, Département de géologie, Unité de recherche géotechnique \\ environnementale et matériaux civils, Sfax, Tunis \\ ${ }^{2}$ Université de Sfax, Faculté des sciences, Département de géologie, Unité de recherche géotechnique environnementale \\ et matériaux civils, Sfax, Tunis
}

\begin{abstract}
Résumé. Les déchets industriels et particulièrement les déchets liquides, renferment dans la majorité des cas des éléments dangereux et toxiques pouvant causer des dégâts environnementaux qui mettent en péril la santé des populations s'ils sont déversés directement dans les réseaux d'assainissement. L'utilisation de ces déchets liquides comme eau de gâchage des liants hydrauliques constitue la méthode la plus utilisée pour réduire l'impact négatif de ces déchets sur l'environnement. Différents échantillons de déchets liquides générés des ateliers mécaniques industriels et chargés en métaux lourds ont été prélevés et analysés. Ces échantillons diffèrent par leurs concentrations en métaux lourds et par leur $\mathrm{pH}$ (eaux acide et basique). La nature de ces liquides influence les propriétés rhéologiques et mécaniques des mortiers préparés. De ce fait, une étude d'approche a été adoptée pour sélectionner les meilleures formulations susceptibles de piéger les métaux lourds du déchet dans la matrice cimentaire des mortiers tout en assurant une bonne rhéologie et de bonnes résistances mécaniques.

A travers les résultats des essais mécaniques, des essais de lixiviations, et des observations microscopiques, une bonne rétention des métaux lourds dans le mortier a été remarquée à court terme.

Mots-clefs: déchet, matrice cimentaire, métaux lourds, essai mécanique, lixiviation, observation microscopique.
\end{abstract}

\section{Introduction}

Les unités de traitement des métaux engendrent une pollution minérale toxique aux cours des opérations de dégraissage et de décharge des pièces métalliques avant usinage, et de traitement chimique. Le potentiel de toxicité de ces déchets dépend en grande partie de la façon dont ils sont éliminés et traités. En Tunisie, Le nombre estimatif des industries de traitement de surface est de 42 [1]. On cite par exemple les ateliers mécaniques industriels dont les déchets liquides générés font objet de cette étude. L'activité de traitement de surface regroupe tous les procédés chimiques et électrochimiques de modifications des propriétés de la surface de pièces le plus souvent métalliques [2]. Durant toutes les opérations de traitement comme le chromage, les pièces sont trempées dans des bains de solution aqueuse puis dans des cuves de rinçage à l'eau, permettant d'arrêter les réactions chimiques à la surface des pièces et d'éviter la contamination d'un bain par le précédent. Les eaux de rinçage et surtout les bains sont de nature fortement chargés en divers espèces et particulièrement en métaux lourds (Fe, $\mathrm{Zn}, \mathrm{Cr}, \mathrm{Cu}, \mathrm{Ni} . .$.$) [1]. Cette grande quantité$ de solutions liquides chargées en métaux lourds est ensuite transformée au sein de l'industrie en boues contaminées. Ces dernières représentent à elles seules $68 \%$ de la quantité totale des déchets industrielles spéciaux par an [3]. De plus, Les normes environnementales actuelles exigent le traitement de ces métaux lourds. Ainsi que partout dans le monde, les décisions politiques ont tranché pour diminuer les volumes de déchets à stocker; ils doivent être limités au strict minimum [3]. D'où l'idée d'utilisation de ces déchets liquides, avant leurs transformations en boue, comme eau de gâchage des liants hydrauliques. La facilité de mise en œuvre du procédé de Stabilisation/Solidification par le liant hydraulique et son faible coût justifient qu'il soit actuellement le plus utilisé parmi les différents procédés de stabilisation existants [4]. Le liant le plus communément utilisé pour contenir ces métaux lourds est le ciment Portland. La contenance de ces métaux lourds varie entre la fixation chimique et le piégeage physique pour produire une matrice ciment/déchet stable. Le but de cette étude est de déterminer les formulations de mortier; à base de ciment Portland; les plus efficaces pour le piégeage à court terme 
des métaux lourds des eaux résiduaires des ateliers mécaniques industriels. Ainsi que d'étudier les modes de piégeage de ces métaux lourds dans la matrice de ciment hydratée.

\section{Matériaux et méthodes}

\subsection{Sélections des matériaux}

Pour atteindre les objectifs visés, un ciment de type HRS I 42,5 N, un plastifiant réducteur d'eau « PL », du sable et deux types d'eau de gâchage ont été utilisés. Le sable utilisé est un sable quartzeux, lavé, criblé et traité dont les grains s'échelonnent de 0 microns à $2 \mathrm{~mm}$. Les deux types d'eau de gâchage sont : l'eau potable du robinet et les déchets liquides riches en métaux lourds.

\subsection{Méthodes d'essais}

Des travaux préliminaires de caractérisation chimique des échantillons de déchets liquides et de détermination du dosage optimal d'adjuvant considéré pour le ciment HRS I ont été entrepris. Le dosage du rapport entre le ciment et le sable est en général volumétrique de $1 / 3$ et le rapport de l'eau sur ciment est environ 0,35. Par ailleurs, toutes les formulations de mortiers préparées sont composées en poids d'une partie de ciment et de 3 parties de sable. Tous les mortiers sont confectionnés selon la norme NBN EN 1015-2. Mais ils diffèrent par: la nature de l'eau de gâchage (eau de robinet ou solution de déchet liquide), le rapport $\mathrm{E} / \mathrm{C}$ ou déchet/ciment $(\mathrm{D} / \mathrm{C})$ et par l'ajout d'adjuvant. On a adopté la norme NBN EN 1015-3 pour la détermination de la consistance du mortier frais. La table à secousse utilisée est de model E086, qui est conforme à la norme ASTM C230 BS4551 .

Soixante huit mélanges de mortiers ont été préparés avec : un ciment type I à haute résistance au sulfate, à base de l'eau de robinet ou du déchet liquide et avec ou sans adjuvant. Ils différent aussi par leurs rapports $\mathrm{E} / \mathrm{C}$ ou déchet/ciment $(\mathrm{D} / \mathrm{C})$ de 0,3 à 0,65 . Ces mortiers ont fait l'objet d'une caractérisation rhéologique en mesurant leurs consistances avec la table à secousse. L'approche méthodologique adoptée pour la détermination des formulations de mortiers les plus efficaces pour le piégeage des métaux lourds à court terme est basée dans un premier temps, sur la détermination des résistances de différents mortiers témoins (préparés à base d'eau de robinet). Dans un deuxième temps, de choisir les formulations de mortier (à base de déchet) ayant les mêmes consistances que les mortiers témoins les plus résistants. Ces formulations sélectionnées ont fait l'objet d'une étude de toxicité et d'une étude microstructurale par observation au microscope électronique à balayage (MEB). La caractérisation mécanique des différents mortiers a été réalisée après 28 jours de prise sur des échantillons prismatiques de taille $4 * 4 * 16 \mathrm{~cm}$ à l'aide d'une presse tri axial. L'étude de la toxicité est déterminée par l'essai de lixiviation dans l'eau déminéralisé après 28 jours de prise. La lixiviation est réalisée selon la norme française AFNOR X 31-211 sur des éprouvettes cylindriques de mortiers $(\varnothing=4 \mathrm{~cm}, \mathrm{~h}=8$ $\mathrm{cm}$ ) et sous une agitation de 24 heures "figure 1". Puis les lixiviats sont filtrés et analysés chimiquement par spectroscopie par absorption atomique.

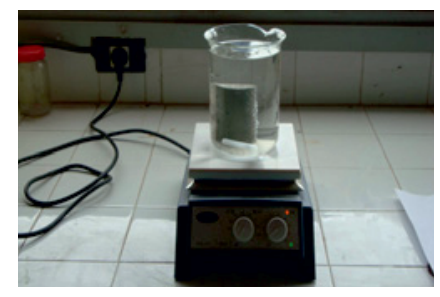

Fig. 1. Dispositif de l'essai de lixiviation

\section{Présentation et analyse des résultats expérimentaux}

\subsection{Caractérisation des déchets}

L'analyse chimique par spectroscopie par absorption atomique des différents échantillons de déchets liquides prélevés des ateliers mécaniques industriels montrent que leurs compositions chimiques diffèrent de jour en jour et que ces derniers présentent des concentrations élevées en métaux lourds [Tableau 1]. Les plus abondants sont: Zn, $\mathrm{Cr}, \mathrm{Mn}$ et $\mathrm{Fe}$. Les concentrations de ces métaux dépassent parfois les limites fixées par la norme NT 106.002 [5]. Les valeurs de métaux lourds qui dépassent les limites fixées par cette norme sont représentées dans le tableau 1 en gras. La mesure du $\mathrm{pH}$ des déchets prélevés permet d'en distinguer trois classes (acide, proche du neutre et basique). Trois échantillons de déchets (D1, D2 et D3) sont sélectionnés (selon le critère de $\mathrm{pH}$ ) et prélevés pour être testés [Tableau 1]

\begin{tabular}{|c|c|c|c|c|c|}
\hline \multicolumn{2}{|l|}{ Déchet } & D1 & D2 & D3 & $\begin{array}{c}\text { Valeurs } \\
\text { limites } \\
\text { Norme } \\
\text { NT } \\
106.002 \\
(\mathrm{mg} / \mathrm{l})\end{array}$ \\
\hline \multicolumn{2}{|c|}{$\mathrm{pH}$} & 4,5 & 6 & 11 & \\
\hline \multirow{7}{*}{ 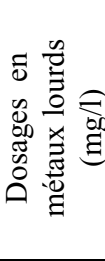 } & $\mathrm{Zn}$ & 2,25 & 1,21 & 149,45 & 5 \\
\hline & $\mathrm{Cr}$ & 0,33 & 2,026 & 0,31 & 0,5 \\
\hline & $\mathrm{Mn}$ & 0,0076 & 0,0136 & 0,32 & 0,5 \\
\hline & $\mathrm{Pb}$ & 0,0729 & 0,0887 & 0,0479 & 0,1 \\
\hline & $\mathrm{Fe}$ & $<0,006$ & 1,8 & 0,03 & 1 \\
\hline & $\mathrm{Ni}$ & $<0,01$ & 1,803 & $<0,01$ & 0,2 \\
\hline & $\mathrm{Cd}$ & \multicolumn{3}{|c|}{$<0,005$} & 0,5 \\
\hline
\end{tabular}

Tableau 1. Caractérisation chimique des déchets liquides

\subsection{Etude des mortiers}

\subsubsection{Etude de la maniabilité des mortiers}


Les résultats de consistances des mortiers frais, effectués sur soixante huit formulations de mortier en appliquant la norme NBN EN 1015-3, sont présentés à la figure2.

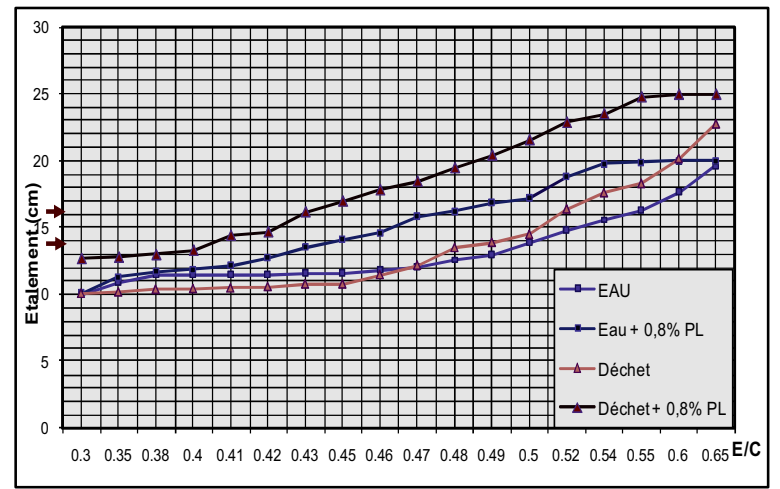

Fig.2.Variation de la consistance des mortiers en fonction de la nature de l'eau de gâchage.

Généralement, On a remarqué au cours de la préparation des mortiers que les mélanges ayant les rapports $\mathrm{E} / \mathrm{C}$ ou $\mathrm{D} / \mathrm{C}<0,45$ sont non maniables et très visqueux et au cours du durcissement, ils ont des mauvaises structures.

\subsubsection{Caractérisation mécanique des mortiers et sélection de leurs formulations}

Cinq formulations de mortiers témoins (à base d'eau de robinet) dont les rapports $\mathrm{E} / \mathrm{C}$ varient entre 0,45 et 0,65 ont été préparées sous formes d'échantillons prismatiques pour évaluer leurs résistances " figure 3 "

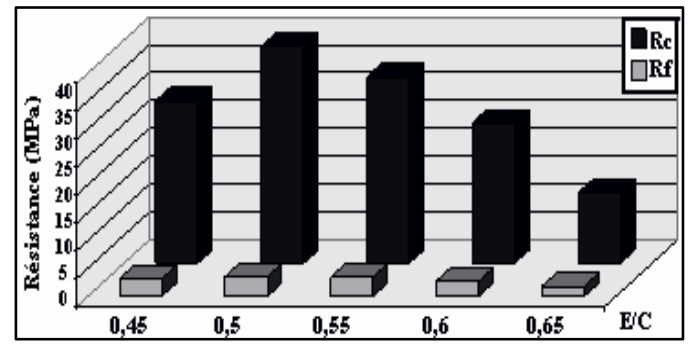

Fig.3. Evolution de la résistance des mortiers témoins en fonction du rapport $\mathrm{E} / \mathrm{C}$ (Rc: résistance à la compression, $\mathrm{Rf}$ : résistance à la flexion)

L'observation des résultats de résistance à la compression et à la traction des différentes formulations de mortiers cités dans la figure 4 montre que les mortiers témoins les plus résistants correspondent aux rapports $\mathrm{E} / \mathrm{C} 0,5$ et 0,55 . L'étalement de ces deux mortiers a été respectivement de l'ordre de 13,9 et $16,3 \mathrm{~cm}$.

En se référant à la figure 3 , les mortiers ayant les mêmes consistances que ces deux derniers rapports ont été sélectionnés [tableau 2].

\begin{tabular}{|c|c|c|c|c|}
\hline $\begin{array}{c}\text { Nature de } \\
\text { liquide } \\
\text { Etalement }\end{array}$ & Eau & Déchet & $\begin{array}{c}\text { Eau+ } \\
0,8 \% \mathrm{PL}\end{array}$ & $\begin{array}{c}\text { Déchet }+ \\
0,8 \% \mathrm{PL}\end{array}$ \\
\hline $13,9 \mathrm{~cm}$ & $\mathrm{E} / \mathrm{C}=0,5$ & $\mathrm{D} / \mathrm{C}=0,49$ & $\mathrm{E} / \mathrm{C}=0,45$ & $\mathrm{D} / \mathrm{C}=0,41$ \\
\hline $16,3 \mathrm{~cm}$ & $\mathrm{E} / \mathrm{C}=0,55$ & $\mathrm{D} / \mathrm{C}=0,52$ & $\mathrm{E} / \mathrm{C}=0,48$ & $\mathrm{D} / \mathrm{C}=0,43$ \\
\hline
\end{tabular}

Tableau 2. Formulations des mortiers sélectionnés
Ces différentes formulations de mortiers sélectionnées ont été préparées sous formes d'échantillons prismatiques en utilisant les trois types de déchets (D1, D2 et D3) puis testés après 28 jours par l'essai de résistance à la compression et à la flexion. Les résultats sont figurés sous formes d'histogrammes "figure 4 et 5 ".
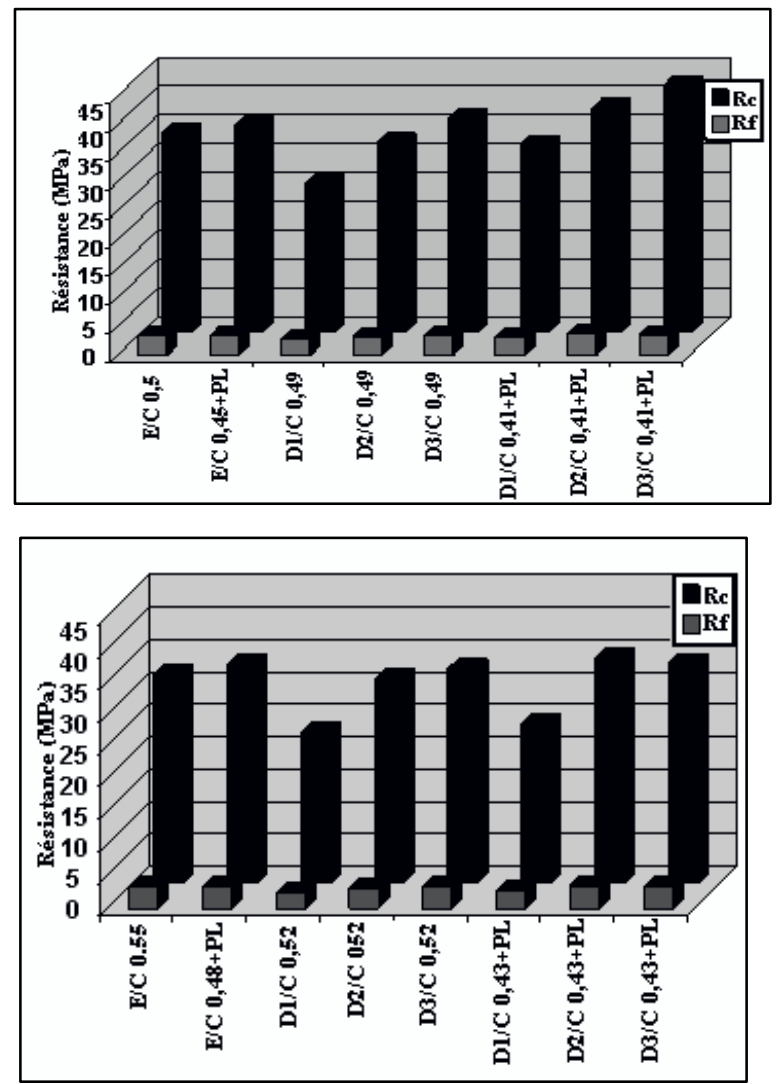

Fig.4. Résistance des mortiers ayant la même consistance que le mortier témoin $\mathrm{E} / \mathrm{C}=0,5$ (avec $\mathrm{E} / \mathrm{C}:$ eau/ciment, et $\mathrm{D} / \mathrm{C}:$ déchet/ciment)

Fig.5. Résistance des mortiers ayant la même consistance que le mortier témoin $\mathrm{E} / \mathrm{C}=0,55$

L'observation de ces différentes valeurs montre que :

- Les mortiers confectionnés par le déchet D1 ou D2 développent des résistances inférieures à celle du mortier témoin et cela avec tous les rapports $\mathrm{D} / \mathrm{C}$ en absence d'adjuvant. En effet, les résistances des mortiers non adjuvantés, de rapports $\mathrm{D} / \mathrm{C}=0,49$ et confectionnés avec D1 ou D2 représentent respectivement $74,7 \%$ et $94,8 \%$ de celle du mortier témoin.

-En absence d'adjuvant, les meilleures valeurs de résistance correspondent aux mélanges préparés avec D3 dont elles dépassent même les résistances des échantillons témoins. Ceci est bien détecté avec $\mathrm{D} / \mathrm{C}=0,49$ et expliqué par l'augmentation du $\mathrm{pH}$ de la matrice cimentaire sous l'effet de la forte basicité du déchet D3.

- L'utilisation d'adjuvant réducteur d'eau améliore fortement la résistance mécanique à 28 jours, surtout en cas d'utilisation du déchet D2 ou D3. Elles dépassent 
celles des échantillons témoins adjuvantés (de rapport $\mathrm{D} / \mathrm{C} 0,41)$ respectivement de $8,3 \%$ et de $19,4 \%$ du rapport.

\subsubsection{Evaluation de la toxicité}

Des échantillons cylindriques $(\mathrm{h}=8 \mathrm{~cm}$ et $\emptyset=4 \mathrm{~cm})$ des mortiers sélectionnés [tableau 2] ont été préparés et testés après 28 jours par l'essai de lixiviation dans l'eau déminéralisée selon la norme X31-211 "figures 6 ”. Les résultats sont représentés dans le tableau 3.

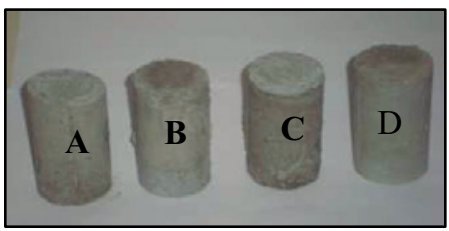

Fig. 6. Photos de 4 échantillons de mortiers (A: témoin, $\mathrm{B}$ : mortier préparé avec D1, C: mortier préparé avec D2, D: mortier préparé avec D3)

Tableau 3. Pourcentages de piégeage des métaux lourds dans les différents mortiers

\begin{tabular}{|c|c|c|c|c|c|}
\hline \multirow{2}{*}{\multicolumn{2}{|c|}{$\begin{array}{l}\text { Métaux teurds } \\
\text { Mortiers } \\
\text { Mortiers } \\
\text { témoins: } \\
\text { Avec ou sans } \\
\text { adjuvant } \\
(\text { E } / \mathrm{C}=0,5 \text { et } \\
0,55) \\
\end{array}$}} & $\mathrm{Zn}$ & $\mathrm{Fe}$ & Mn & $\begin{array}{l}\mathrm{Cr}, \mathrm{Cu}, \\
\mathrm{Pb}, \mathrm{Ni}, \\
\mathrm{Cd}, \mathrm{Co} \\
\end{array}$ \\
\hline & & \multicolumn{4}{|c|}{ Absence de métaux lourds $<0,01 \mathrm{mg} / 1$} \\
\hline \multirow{3}{*}{$\begin{array}{l}\text { Déchet } \\
D / C=0,4 \\
9\end{array}$} & D1 & $100 \%$ & $93 \%$ & $84 \%$ & \multirow{3}{*}{$100 \%$} \\
\hline & D2 & $86 \%$ & $100 \%$ & $75 \%$ & \\
\hline & D3 & $100 \%$ & $100 \%$ & $100 \%$ & \\
\hline \multirow{3}{*}{$\begin{array}{l}\text { Déchet } \\
D / C=0,5 \\
2\end{array}$} & D1 & $99 \%$ & $90 \%$ & $71 \%$ & \multirow{3}{*}{$100 \%$} \\
\hline & $\mathrm{D} 2$ & $85 \%$ & $99 \%$ & $73 \%$ & \\
\hline & D3 & $100 \%$ & $99 \%$ & $73 \%$ & \\
\hline \multirow{3}{*}{$\begin{array}{l}\text { Déchet+ } \\
0,8 \% \text { PL } \\
(\mathrm{D} / \mathrm{C}= \\
0,41 \text { ou } \\
0,43)\end{array}$} & D1 & $100 \%$ & $100 \%$ & $100 \%$ & \multirow{3}{*}{$100 \%$} \\
\hline & D2 & $100 \%$ & $100 \%$ & $100 \%$ & \\
\hline & D3 & $100 \%$ & $100 \%$ & $100 \%$ & \\
\hline
\end{tabular}

L'analyse des lixiviats montre que :

$>$ Les valeurs des métaux lourds détectés dans certains lixiviats sont très inférieures aux limites fixées par les normes tunisiennes de rejets liquides NT 106.002 [5]

$>$ le piégeage de divers métaux est très efficace comme le fer, chrome, plomb, cuivre, nickel et le cobalt dans toutes les formulations de mortiers et avec n'importe quel type de déchet (D1, D2 ou D3). Alors que pour d'autres comme $\mathrm{Zn}$ et $\mathrm{Mn}$, leur rejet dépend de la formulation du mortier et surtout du $\mathrm{pH}$ du déchet utilisé. En effet, Ces rejets sont favorisés par l'acidité du déchet. D'ailleurs les métaux risquent de se solubiliser dans un milieu acide, ils peuvent former des hydroxydes métalliques dans un milieu alcalin [6]

$>$ L'utilisation de l'adjuvant $(0,8 \%)$ permet de maintenir davantage les métaux lourds au sein de la matrice cimentaire (100\% piégeage).

\subsubsection{Etude de la microstructure de la matrice cimentaire du mortier par MEB}

Les résultats d'analyse de la microstructure par MEB des mortiers sélectionnés sont groupés dans les figures 7 , 8,9 et 10 .

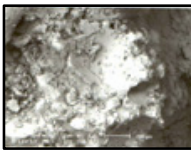

A

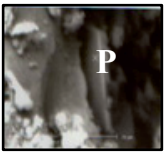

B

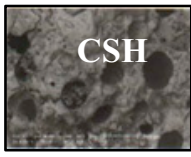

C
Fig.7. Microstructures des mortiers témoins (A : en absence d'adjuvant ; B et C : en présence d'adjuvant, $\mathrm{P}$ : Portlandite, CSH: Silicate de calcium hydraté)
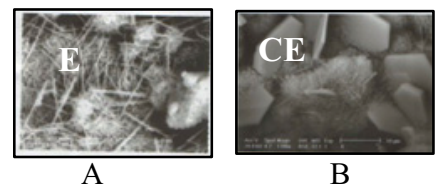

Fig.8. Microstructures des mortiers préparés avec D1 (A: en absence d'adjuvant ; B : en présence d'adjuvant, E: Ettringite, CE: Chromo-ettringite)

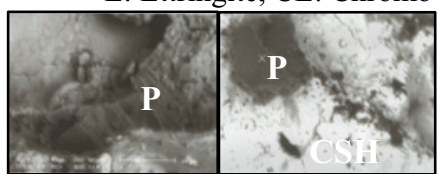

A

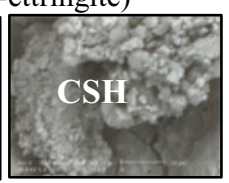

$\mathrm{C}$
Fig.9. Microstructures des mortiers préparés avec D2

(A : en absence d'adjuvant ; B et C: en présence d'adjuvant, $\mathrm{P}$ : Portlandite, CSH: Silicate de calcium hydraté)

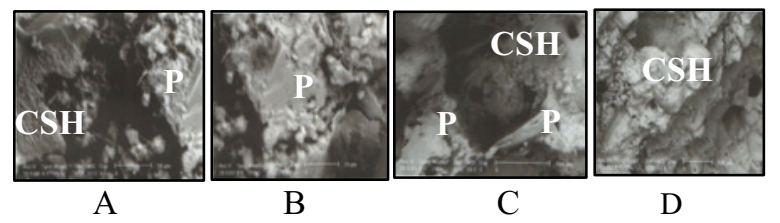

Fig. 10. Microstructures des mortiers préparés avec D3 (A, B : en absence d'adjuvant; $\mathrm{C}$ et $\mathrm{D}$ : en présence d'adjuvant,

P: Portlandite, CSH: Silicate de calcium hydraté)

D'après la figure8, on remarque que l'utilisation $\mathrm{du}$ plastifiant dans le mortier témoin améliore l'hydratation de la matrice cimentaire par formation des cristaux de Portlandites et du gel de C-S-H. L'utilisation du déchet D1 entraîne une formation excessive d'Ettringites "figure 9A". Cette observation peut s'expliquer par l'acidité du déchet qui diminue le $\mathrm{pH}$ de la matrice cimentaire et favorise la formation d'aiguille d'ettringite. L'ajout du plastifiant à ce type de déchet, favorise le piégeage du chrome par formation des cristaux hexagonaux de chromo-ettringite. De plus, ces cristaux sont entourés par une structure combinée de gel de $\mathrm{CSH}$ et des aiguilles d'Ettringite "figures 9B". En effet, le chromo-ettringite 
se forme par substitution du Cr IV par (SO4) ${ }_{2}^{-}$[7]. De plus, la microanalyse montre l'absence totale du chrome avec piégeage surtout du plomb, cadmium et zinc dans le gel de CSH et d'aiguilles d'Ettringite "figure 11".

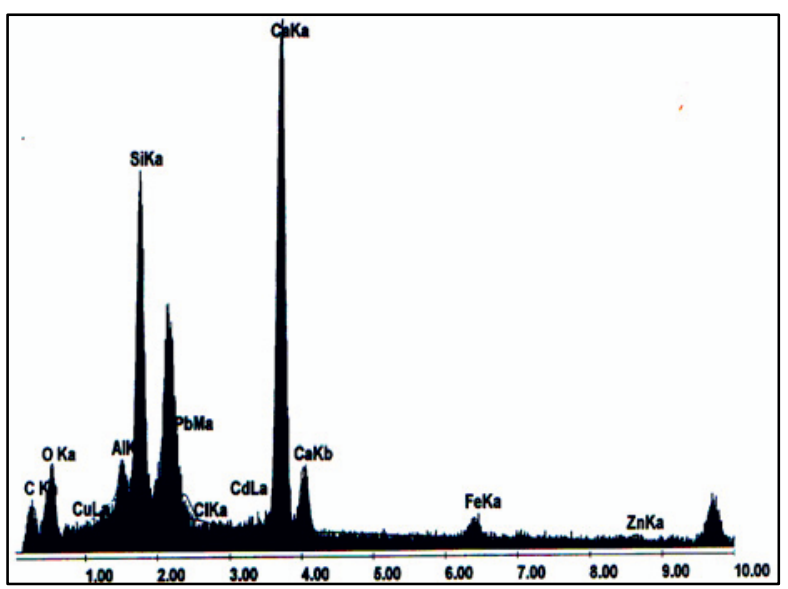

Fig. 11. Microanalyse du gel de $\mathrm{CSH}$ et des aiguilles d'ettringite du mortier préparé avec D1.

L'utilisation de D2 ou D3 favorise principalement la formation des cristaux de Portlandite et de CSH "figures 10 et 11 ". En ajoutant l'adjuvant, une augmentation de la formation du $\mathrm{CSH}$ au profit de la portlandite est remarquée. Le CSH est mal cristallisé et se présente sous forme d'amas granulaire et de fibre "figure 11, photo $\mathrm{C}$ et D". Les résultats de la microanalyse ont révélés des différences de comportements de piégeage des métaux lourds "figure 13". En effet, certains sont retenus uniquement dans la matrice de $\mathrm{CSH}$ et on ne les détecte pas dans la structure du Portlandite; c'est le cas du chrome et du plomb. D'autres sont piégés dans les deux structures: Portlandite et $\mathrm{CSH}$ comme $\mathrm{Cu}$ et $\mathrm{Cd}$. Alors que d'autres sont piégés surtout dans la structure du $\mathrm{CSH}$, c'est le cas surtout du fer et du zinc "figure 12".

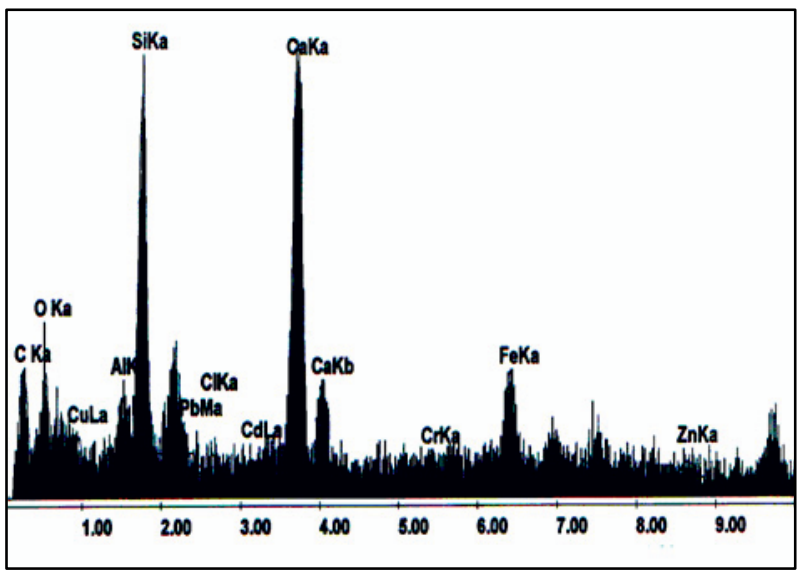

Fig.12. Microanalyse de CSH du mortier préparé avec le déchet D3

\section{Conclusion}

A la lumière de ce qui précède, on peut conclure que :

$>\mathrm{La}$ formation des hydrates dépend de la formulation du mortier et du $\mathrm{pH}$ du déchet.

$>$ L'observation par MEB de la microstructure montrent l'abondance de CSH par rapport à la Portlandite et l'Ettringite dans les mortiers préparés avec le déchet à $\mathrm{pH}$ proche du neutre ou basique et surtout en présence d'adjuvant; Ces résultats sont encourageants puisque:

- La structure CSH capable de piéger les cations métalliques [7]. De plus, les métaux les plus abondants dans le déchet sont des cations ( $\mathrm{Zn}, \mathrm{Cr}, \mathrm{Mn}$ et Fe...)

- Le CSH participe le plus au développement de la résistance du matériau et assure l'essentiel de la cohésion de ce dernier et de sa durabilité.

$>$ L'utilisation d'adjuvant dans les mortiers préparés à base de déchet contribue positivement à la fixation de la majorité des métaux lourds au sein de la matrice cimentaire du mortier tout en améliorant les performances mécaniques.

$>$ L'observation des résultats de: résistance à la compression et à la flexion, de lixiviation et de la microstructure nous permet de conclure que les déchets liquides testés (à $\mathrm{pH}$ basique ou proche du $\mathrm{pH}$ neutre) peuvent être utilisé comme eau de gâchage de la matrice cimentaire du mortier à un rapport déchet/ciment de 0,41 tout en lui ajoutant $0,8 \%$ d'adjuvant réducteur d'eau.

\section{References}

1. M. Keskes. Eude du comportement des boues industrielles contaminées stabilisées et solidifiées par des liants hydrauliques (cas de l'usine AMI à sfax). Thèse de doctorat en sciences géologiques, Université de Sfax, Département de géologie, Faculté des sciences, 13-14 (2010)

2. J.N.Diet, P. Mosczkowicz, N. Alain, Solidification de boues d'hydroxydes métalliques par des ciments Portland: comportement du ciment en présence de boues d'hydroxydes de chrome. Actes de proceedings Stabilisation des déchets \& environnement,13-16 Avril, 264 (1999)

3. M. Choura, M. Keskes, M.J, Rouis, Traitement par stabilisation/solidification à l'aide de liants hydrauliques des boues issus des industries de traitement de surface par galvanoplastie, Journal déchet sciences \& techniques, revue Francophone d'écologie industrielle- $\mathrm{N}^{\circ} 37-1^{\text {er }}$ trimestre (2005)

4. C. Fantozzi-Merle, Etude de matériaux à base de liants hydraulique contenant des polluants organiques modèles: propriétés structurales et de transfert. Thèse en sciences et techniques du déchet. Institut National des sciences appliquées de Lyon, 24-25 (2003)

5. Norme tunisienne, rejets d'effluents dans le milieu Hydrique, N.T.106.002, 62-65 (1989)

6. H. Hammi, la pollution des eaux par les métaux lourds, les IIIème olympiades tunisiennes de chimie, 13 (2010)

7. E. Moudilou, Cinétiques et mécanismes de relargage des métaux lourds présents en traces dans les matrices cimentaires. Thèse, Université d'Orléans, 41 (2000) 